\title{
Discours
}

Revue de linguistique, psycholinguistique et

informatique. A journal of linguistics, psycholinguistics and computational linguistics

$27 \mid 2020$

Varia

\section{De la mention de l'individu à celle du groupe : usage des expressions référentielles en fusion et en partition dans les récits d'enfants de 5 à 8 ans}

Camille Dupret

\section{OpenEdition}

Journals

Édition électronique

URL : http://journals.openedition.org/discours/11005

DOI : 10.4000/discours. 11005

ISSN : 1963-1723

Éditeur :

Laboratoire LATTICE, Presses universitaires de Caen

\section{Référence électronique}

Camille Dupret, « De la mention de l'individu à celle du groupe : usage des expressions référentielles en fusion et en partition dans les récits d'enfants de 5 à 8 ans ", Discours [En ligne], 27 | 2020, mis en ligne le 23 décembre 2020, consulté le 13 avril 2021. URL : http://journals.openedition.org/discours/11005 ; DOI : https://doi.org/10.4000/discours. 11005 

Revue de linguistique, psycholinguistique et informatique

De la mention de l'individu à celle du groupe: usage des expressions référentielles en fusion et en partition dans les récits d'enfants de 5 à 8 ans

Camille Dupret

CLESTHIA - Langage, systèmes, discours

Université Sorbonne Nouvelle - Paris 3

Camille Dupret, «De la mention de l'individu à celle du groupe: usage des expressions référentielles en fusion et en partition dans les récits d'enfants de 5 à 8 ans », Discours [En ligne], 27 | 2020, mis en ligne le 23 décembre 2020.

URL: http://journals.openedition.org/discours/11005

Titre du numéro: Varia

Coordination: Shirley Carter-Thomas \& Frédéric Landragin

Date de réception de l'article: 15/06/2020

Date d'acceptation de l'article: 18/09/2020 



\title{
De la mention de l'individu à celle du groupe : usage des expressions référentielles en fusion et en partition dans les récits d'enfants de 5 à 8 ans
}

\author{
Camille Dupret \\ CLESTHIA - Langage, systèmes, discours \\ Université Sorbonne Nouvelle - Paris 3
}

\begin{abstract}
Cette étude s'intéresse à l'usage des expressions référentielles par les enfants en récit quand ils procèdent à un regroupement de référents (fusion) et quand ils mentionnent séparément les référents d'une entité précédemment présentée comme un groupe (partition). Les enfants ayant des difficultés à faire un usage clair de la référence en récit quand plusieurs référents sont candidats à être sélectionnés en discours (Hickmann, 2002), cette étude vise à observer comment ils expriment la relation entre une expression référentielle au pluriel et ses antécédents dispersés dans la chaîne discursive et, à l'inverse, les formes qu'ils utilisent pour séparer plusieurs référents précédemment mentionnés comme un groupe. 45 enfants âgés de 5 à 8 ans ont été enregistrés en train de produire deux récits à partir de supports imagés sans texte. Les résultats montrent que les enfants utilisent majoritairement des formes pronominales lors des fusions, peu importe la distance par rapport à l'antécédent, indiquant que les fusions s'inscrivent dans la continuité des chaînes référentielles formées par les antécédents. Cependant, les plus grands (de 7-8 ans) semblent être davantage capables de se reposer sur l'univers discursif institué pour clarifier les antécédents des expressions référentielles au pluriel. En partition, on trouve une plus grande variété de formes fortes qui expriment un contraste avec l'antécédent, mais les enfants les plus grands sont plus sensibles à la distance par rapport à l'antécédent dans le choix des formes.
\end{abstract}

Mots clés: référence, groupement de référents, récit, enfants, acquisition / développement

This study investigates children's use of referring expressions in narratives when they express a referents grouping (i.e., fusion) and mention separate referents of an entity previously presented as a group (i.e., partition). As children seem to struggle to make a clear use of reference in narratives when different referents are candidate to be selected in discourse (Hickmann, 2002), this study aims to determine how they express the relations between a plural referring expression and its split antecedents within the discursive chain and, on the other hand, the forms they use to split several referents previously mentioned as a group. 45 children aged from 5 to 8 were video-recorded while recounting two different stories from textless sets of pictures. Results show that children use mostly pronominal forms in referents grouping, regardless of the distance of the antecedent, suggesting that referents grouping stands in continuity with the referential chains formed by the antecedents. However, older children of 7-8 years old seem to better use the established discursive universe to clarify the antecedents of plural referring expressions. In referents splitting, there is a greater variety of strong forms that express a contrast to the antecedents, but older children are more sensitive to distance from the antecedent in the choice of forms.

Keywords: reference, referents grouping, narrative, children, acquisition/development 


\section{Introduction}

Le développement de la référence chez l'enfant constitue un processus complexe adoptant des trajectoires différentes selon les types de discours (Serratrice et Allen, 2015). Dans les discours narratifs, l'enjeu pour l'enfant consiste en partie à "choisir»' des formes linguistiques adaptées à la présentation d'informations nouvelles tout en gérant le maintien d'informations préalablement mentionnées pour assurer la cohérence du récit (Fayol, 1983 et 2000). En cela, la référence aux différentes entités impliquées dans la dynamique du récit est cruciale pour que celui-ci soit interprétable par l'interlocuteur. La production d'un récit constitue ainsi une activité complexe pour l'enfant qui n'acquiert un usage des expressions référentielles proche de celui de l'adulte qu'aux alentours de 8 voire 10 ans selon les recherches et les critères de performance (Bamberg, 1986; Berman et Slobin, 1994; De Weck et Salazar Orvig, 2014; Hickmann, 2002; Karmiloff-Smith, 1981). Avant cela, il présente certaines difficultés à faire un usage non ambigu des expressions référentielles, en particulier dans les contextes où plusieurs référents partageant des traits communs (animéité, genre grammatical notamment) sont candidats à être sélectionnés dans le discours (Karmiloff-Smith, 1985; Serratrice, 2013) et se retrouvent de ce fait, en concurrence (Arnold et Griffin, 2007).

Ainsi, d'un côté l'enfant commence à maitriser les principes de l'usage des expressions référentielles assez tôt en dialogue (Allen et al., 2015; Salazar Orvig et al., 2010) mais présente un certain décalage à faire de même dans des séquences discursives plus longues comme les récits (Hickmann et al., 2015). De la même manière, au sein des narrations enfantines se mêlent des marques de difficultés à introduire et maintenir clairement les entités dans le récit (Aksu-Koç et Nicolopoulou, 2015; Hickmann, 2002; Jisa, 2000; Karmiloff-Smith, 1985), et des conduites complexes et diversifiées qui donnent parfois l'impression d'une certaine maitrise de l'organisation discursive. Il est difficile de déterminer si la diversité de ces conduites constitue de réelles stratégies de l'enfant qui les utilise pour leur fonction discursive ou si elles sont des marques de transition et de l'acquisition progressive de nouveaux usages. Parmi ces phénomènes, le fait de passer de plusieurs référents animés mentionnés séparément à la mention d'un groupe par le biais d'une expression référentielle au pluriel a pour effet de neutraliser la concurrence entre les référents regroupés, limitant ainsi les difficultés susmentionnées qui impactent la clarté des récits. Ce phénomène est illustré dans l'exemple $[1]^{2}$ avec les éléments en gras.

[1] Eva, 7;07

ENF2 là c'est un ours et un lapin qui sont bien tranquilles.

ENF3 et le renard $\mathrm{i}(\mathrm{l})$ s'est caché parc(e) qu'il a envie $\mathrm{d}(\mathrm{e})$ prendre des poules.

1. La notion de "choix» est à nuancer dans la mesure où l'enfant n'a pas conscience de sélectionner une forme parmi les possibilités d'exploitation de l'axe paradigmatique qui s'offrent à lui.

2. La transcription des exemples de cet article reprend les énoncés des enfants comme ils ont été produits, restituant ainsi les éventuelles formes non standard utilisées. 
ADU5 mh-mh.

ENF4 et là le renard $\mathrm{i}(\mathrm{l})$ vient $\mathrm{d}(\mathrm{e})$ prendre une poule et euh les animaux sont en train $\mathrm{d}(\mathrm{e})$ dire $<$ reviens reviens $>$ [ $=$ ! discours rapporté] pour euh rendre la poule.

Cependant, ce procédé implique d'autres difficultés quant au choix de la forme pour exprimer la continuité entre la mention des référents seuls et le groupe tout en tenant compte de la concurrence éventuelle avec d'autres chaînes référentielles pour que l'interlocuteur identifie qui a été regroupé par l'expression référentielle au pluriel. Ici, l'enfant utilise habilement un syntagme nominal («les animaux») pour fusionner les référents «un ours» et «un lapin» exprimant ainsi un contraste avec les deux autres référents («le renard» et «une poule») qui apparaissent en concurrence en ENF4. D'autre part, l'existence de groupes dans la progression du récit entraîne parfois la dissociation des individus à d'autres moments du récit, c'est le cas de l'usage des expressions référentielles en gras en ENF30 dans l'exemple [2].

[2] Hélène, 6;07

ENF27 et le renard $\mathrm{i}(1)$ voit $\mathrm{i}(1)$ monte avec la poule.

ENF28 et eux [//] les [//] eux i $(1)$ s commencent à courir.

\%com: ENF pointe le groupe composé de l'ours du lapin et du coq

ENF29 là i(l)s ont monté.

ENF30 et là le lapin $i(l)$ pousse l'ours pour qu'i(l) rentre pour aller avec la poule.

À nouveau, le choix de la forme pour exprimer l'extraction d'une entité est déterminant pour l'identification des référents dans le récit. Dans l'exemple ci-dessus, l'enfant utilise là aussi des formes fortes («le lapin il» et «l'ours») pour clarifier l'identification des référents qu'elle a dissociés suite à l'expression référentielle au pluriel en ENF29.

\section{Les fusions et les partitions dans la dynamique des chaînes référentielles}

Fusions et partitions relèvent d'un processus particulier de dérivation sur le plan référentiel, cela veut dire que l'introduction d'un nouvel objet de discours ne revêt pas le statut habituel d'une première mention (syntagmes nominaux indéfinis ou noms propres selon les standards de la langue écrite) puisque celui-ci est évoqué comme issu de la mention d'une autre expression référentielle par des indices anaphoriques (Schnedecker et Landragin, 2014). En effet, les processus de dérivation peuvent relever de procédés de type anaphore associative entre la mention d'un ensemble et l'introduction d'une de ses composantes ou d'un de ses traits caractéristiques qui, de fait, n'est pas présenté comme nouveau dans le discours. C'est par exemple le cas dans «j'ai acheté une voiture d'occasion mais le moteur a un problème» où le moteur, composante de la voiture précédemment introduite, est présenté au moyen 
d'un syntagme nominal défini. Dans les partitions étudiées ici, il en va légèrement différemment dans la mesure où ce n'est pas une caractéristique sous-spécifiée d'une entité qui est dissociée mais bien un membre plein et entier d'un ensemble. De même, pour les fusions, il s'agit davantage de référents déjà mentionnés qui sont repris sous une autre forme que de l'introduction d'un nouvel objet de discours dans la continuité énonciative. Cela dit, le phénomène est similaire aux anaphores associatives puisque lors de la fusion ou de la partition, l'expression référentielle découle nécessairement d'une autre entité précédemment énoncée et/ou de l'univers discursif institué.

Pour Schnedecker et Bianco (1995), la fusion repose sur des antécédents soit présentés conjointement comme en [1], les deux référents coordonnés sont alors aussi saillants et accessibles discursivement l'un que l'autre, ce qui en fait de bons candidats à être repris sous la forme d'un pronom personnel au pluriel immédiatement après, ou alors elle repose sur des antécédents dispersés qui se retrouvent de part et d'autre du prédicat verbal. Dans ce dernier cas, l'intervention de divers paramètres locaux en interaction permet de redistribuer l'accessibilité des différents arguments du verbe. Parmi ces paramètres, les énoncés organisés autour de deux référents assurant des rôles sémantiques qui fonctionnent par paires contrastées type agent-patient permettent de rendre aussi saillants les deux éléments et la valeur sémantique du verbe peut également véhiculer un rôle prépondérant à l'objet du verbe, faisant de lui un candidat probable à la reprise pronominale (Schnedecker et Bianco, 1995). Mais les enfants présentant déjà des difficultés au niveau pragmatique à assurer la continuité thématique des chaînes de coréférence parce qu'ils tendent à alterner les entités en position sujet sans utiliser les formes linguistiques exprimant le contraste (Hickmann, 2002; Jisa et al., 2010; Karmiloff-Smith, 1985), on peut se demander à quel moment se développe la maîtrise de procédés discursifs relativement élaborés permettant de regrouper plusieurs référents au sein d'une expression référentielle au pluriel. De façon générale, sans s'avérer totalement obscures, les expressions référentielles au pluriel après avoir introduit plusieurs personnages dans le discours peuvent manquer de clarté quant à la délimitation exacte des membres du groupe, ce qui constitue un problème pour l'interprétation par l'interlocuteur que relève Landragin (2011) concernant l'annotation automatique des chaînes référentielles.

Les partitions procèdent par un mécanisme inversé, à l'instar de l'analyse de Schnedecker (2006) sur les anaphores prédicatives démonstratives, celles-ci présupposent l'existence d'un sous-ensemble au sein d'un ensemble plus vaste. Cela veut dire que la partition repose, certes, sur un antécédent dont elle découle mais, à l'inverse de la fusion, elle ne participe pas à la progression du groupe précédemment mentionné. La partition apparât en rupture avec cet antécédent prédiquant des propriétés nouvelles à un sous-ensemble, voire à chacune des entités d'un ensemble lors des mises en parallèle du type l'un/l'autre. Aussi, dans les récits écrits d'enfants âgés de 9 à 11 ans, les phénomènes de partition sont sujets à des problèmes de confusions et d'ambiguïtés dans la mesure où certains enfants tendent à séparer un référent du groupe par le biais d'un pronom personnel, de fait, on 
ne sait quelle entité a été partitionnée (Peres Bonnemaison, 2018). Ainsi, si les fusions semblent fréquemment apparaître sous la forme pronominale en discours (Landragin, 2011; Schnedecker et Bianco, 1995; Schnedecker et Landragin, 2014), cette conduite est susceptible d'entrâner davantage de problèmes d'interprétation lors des phénomènes de partition.

Ces études portaient sur le versant écrit du langage, on peut donc se demander s'il en va de même du fonctionnement des fusions et des partitions à l'oral. En effet, la langue écrite étant, par définition, un mode de communication censé être interprétable en l'absence du locuteur-scripteur, l'expression des relations de coréférence et d'inférence repose sur des règles de marquage assez rigides et conventionnelles. La multimodalité de l'oral permet aux locuteurs de s'affranchir des règles strictes de l'écrit et la formation des chaînes référentielles en récit est, de ce fait, différente à l'oral et à l'écrit. De plus, les exemples utilisés présentent souvent des modèles dans lesquels la fusion fait immédiatement suite aux antécédents mais l'éventuelle présence de référents en concurrence avec les candidats à la fusion dans le contexte immédiat, comme le renard et la poule en [1], apporte davantage de complexité à l'étude du phénomène dans les récits oraux d'enfants.

À partir de l'observation des formes utilisées pour regrouper plusieurs référents au sein d'une même expression référentielle ou à l'inverse, pour séparer différents référents précédemment mentionnés comme un groupe, cette étude se propose donc d'analyser comment les enfants inscrivent ces formes dans les chaînes référentielles dont elles découlent. L'objectif est ainsi de déterminer le fonctionnement de ces deux phénomènes à l'oral et la façon dont on peut considérer leur position dans la continuité du discours. En d'autres termes, il s'agit de définir si les expressions référentielles en fusion et en partition constituent, pour les enfants, des chaînes référentielles dérivées d'autres chaînes mais disposant d'un fonctionnement autonome ou si elles s'inscrivent dans la progression de chaînes existantes.

\section{Méthodologie}

\subsection{Recueil de données}

\subsubsection{Participants}

Cette recherche regroupe 45 participants répartis sur 3 groupes selon leur âge et leur niveau scolaire, 15 enfants composaient chacun des groupes. Les données ont été recueillies grâce à un protocole expérimental réalisé en deux sessions d'enregistrement espacées de trois mois environ et qui se sont déroulées à l'école ${ }^{3}$ où les enfants étaient scolarisés. La venue de l'expérimentatrice dans les différentes écoles a fait l'objet d'une

3. L'auteure remercie chaleureusement les directeurs et directrices des écoles concernées d'avoir accepté que la recherche se déroule dans leur établissement. Les remerciements vont également aux enseignants et enseignantes qui se sont adaptés au déroulement des passations auprès de leurs élèves et aux parents qui ont accepté que leur enfant prenne part au projet. 
autorisation de l'inspection académique de Paris et la participation des enfants a été individuellement soumise à l'accord des parents. Le premier groupe (G1) rassemblait les enfants les plus jeunes de l'étude scolarisés en dernière année de maternelle lors des enregistrements. Le plus jeune enfant du groupe 1 avait 5;03 lors de la première session alors que le plus âgé en deuxième session avait 6;02. L'âge moyen lors de la première session était ainsi de 5;08 et il était de 5;11 lors de la seconde session. Le deuxième groupe (G2) était composé d'enfants de niveau CP. Le plus jeune lors de la première session de recueil avait 6;03 et le plus grand lors de la seconde session était âgé de 7;03. L'âge moyen était de 6;08 lors de la session 1 et de 6;11 lors de la session 2. Le troisième groupe (G3) se composait des enfants les plus grands de l'étude, ils étaient scolarisés en CE1 et avaient entre 7 et 8 ans au moment du recueil. Le plus jeune lors de la première session avait 7;02 et l'âge moyen était de 7;07 alors que le plus grand avait 8;04 lors de la seconde session et l'âge moyen était de 7;10. L'ensemble de ces enfants étaient francophones, certains étaient issus de milieux plurilingues mais une enquête réalisée auprès des parents a révélé que tous avaient effectué leurs années de maternelle en France et que le français était la langue qu'ils parlaient majoritairement au quotidien. Afin de préserver la confidentialité des participants, les prénoms ont été modifiés.

\subsubsection{Corpus}

Les enfants étaient invités à raconter une histoire à partir d'un support imagé proposé par l'expérimentatrice. Chacun des enfants a produit deux récits différents: le premier à partir d'un écran d'ordinateur alors que le deuxième récit a été obtenu à partir d'un livre ${ }^{4}$. Les deux histoires imagées ne comportaient pas de texte, il n'était donc pas nécessaire de savoir lire pour les raconter. Elles se prêtaient assez bien à la mise en place de fusions et, de fait, de partitions, dans la mesure où elles mettaient en scène des référents animés qui étaient amenés à collaborer à l'accomplissement d'une tâche commune. La première histoire proposée aux enfants correspondait à une planche de bande dessinée extraite d'un album des Triplés (Lambert, 2016). Dans cette courte histoire, on voit d'abord un enfant dans une cuisine en train de regarder la porte du réfrigérateur située en hauteur. Une enfant arrive ensuite pour l'aider à l'atteindre puis un troisième enfant les rejoint et parvient à ouvrir la porte du réfrigérateur. Sur la dernière image, les trois enfants dégustent une glace ensemble. Ainsi, la plupart des participants à l'étude avaient tendance à procéder à des fusions au fur et à mesure que les nouveaux référents intervenaient dans l'histoire. Pour la seconde histoire, le livre Le voleur de poule (Rodriguez, 1993) a été utilisé. Dans cette histoire, un groupe d'animaux composé d'un ours, d'un lapin et d'un coq entreprennent ensemble de poursuivre un renard qui vole une poule au début de l'histoire. Dès lors, les trois animaux à la poursuite du renard faisaient régulièrement l'objet de fusions et de partitions au fil des récits. Cette histoire révélant finalement que le renard n'a pas enlevé la poule pour la manger mais pour s'en faire une amie,

4. Bien que la différence entre les deux supports puisse avoir une incidence sur l'élaboration des récits, cette variable n'a pas été étudiée dans la présente étude. 
ces deux référents étaient également sujets à des fusions. Le protocole était le suivant : l'expérimentatrice demandait à l'enfant de lui raconter l'histoire au fur et à mesure qu'il la découvrait, de la manière qu'il souhaitait et en prenant le temps qu'il voulait pour le faire. De son côté, l'expérimentatrice se contentait d'écouter l'enfant, n'intervenant que pour des confirmations, des encouragements à poursuivre ou des demandes de répétitions. Celle-ci partageait alors le support imagé avec l'enfant, ce qui a pu renforcer le recours à des conduites déictiques pour mentionner les personnages et les groupes. En effet, comme le souligne Charolles (1988), jusqu’à 11 ans et y compris à l'écrit, l'enfant peut présenter des difficultés à se détacher du support imagé lorsqu'on lui demande de produire un récit à partir d'une succession d'images. Des enregistrements vidéo ont été effectués et les récits ont été transcrits via le logiciel CLAN (Computerized Language Analysis) (MacWhinney, 2000).

\subsection{Codage}

L'analyse se limitait à une liste de référents prédéfinie à partir des histoires, les fusions et les partitions relevées ici correspondent donc à un ensemble fini de référents dont la mention est attendue dans tous les récits. Les expressions référentielles utilisées ont d'abord été codées selon leur forme et leur fonction dans le discours. Les formes utilisées ont été regroupées sous cinq catégories: les syntagmes nominaux (abrégés en NOM), les dislocations du nom ou du pronom (DISLO), les structures clivées du type «il y a les animaux qui courent pour attraper le renard» (CLIV), les pronoms personnels (PROPERS) et les autres types de pronoms (AUTPRO). Le codage de la fonction consistait à déterminer si l'expression référentielle apparaissait en position sujet ou non-sujet. Ensuite, les expressions référentielles utilisées ont été identifiées comme relevant, soit de la référenciation première du groupe de référents ou du référent isolé dans le récit (abrégée en RFPR), soit d'une référenciation seconde (abrégée en RFSC).

Enfin, un système de codage a permis d'identifier les caractéristiques de l'antécédent, à savoir la forme et la fonction d'une part, et la distance par rapport à l'expression référentielle étudiée d'autre part. La forme et la fonction étaient codées selon les mêmes principes que l'expression référentielle elle-même et la distance a été codée en trois catégories selon que l'antécédent se trouvait dans le même énoncé, dans l'énoncé précédent ou à plus d'un énoncé d'écart. Pour les fusions qui présentaient des antécédents dispersés dans le fil du discours, c'est systématiquement le plus proche qui a été relevé pour le codage de ses caractéristiques.

À partir de ce système de codage, une catégorisation binaire de la relation à l'antécédent a été effectuée: on a déterminé si l'expression référentielle était discursivement en continuité avec l'antécédent ou en alternance avec celui-ci. Les contextes de continuité correspondaient soit aux situations où la fusion apparaissait dans la même fonction syntaxique et à proximité directe de l'antécédent isolé le plus proche (même énoncé ou énoncé précédent), soit aux situations où la fusion entrainait un changement de fonction par rapport à l'antécédent mais où elle apparaissait immédiatement après lui (c'est-à-dire le fait de passer de la 
position non-sujet postposée au verbe à la fonction sujet préposée d'un énoncé à l'autre). À l'inverse, étaient considérées comme en ALTERNANCE, les fusions qui apparaissaient alors qu'un autre référent animé occupant la même fonction a été mentionné entre l'antécédent et l'expression référentielle en question, ou bien les cas de changement de fonction par rapport à l'antécédent qui n'apparaissaient pas immédiatement après.

\subsection{Analyses}

Les analyses effectuées ont porté exclusivement sur le passage de la mention de la partie au tout (fusion) et sur le passage du tout à la partie (partition). L'éventuel maintien d'une expression référentielle au pluriel à travers les énoncés n'a pas été codé comme une fusion à chaque fois mais constitue simplement le maintien de la focalisation sur la même entité d'un énoncé à l'autre. De même, les phénomènes de partition se limitent à passer de la mention du groupe à un individu, les reprises de l'individu isolé, si elles ne faisaient pas suite à une nouvelle fusion, n'ont pas été relevées pour l'analyse. Il s'agissait donc de comparer les conduites adoptées en fusion et en partition pour envisager les ressemblances et les différences entre ces deux phénomènes du point de vue de la dynamique des chaînes référentielles en discours. Des tests de Kruskal-Wallis ont ensuite permis d'effectuer des comparaisons entre les groupes d'âges.

\section{Résultats}

\subsection{Les fusions}

Pour évaluer le degré d'autonomie ou de dépendance des fusions par rapport aux chaînes référentielles qu'elles regroupent, une première analyse a consisté à observer si les enfants utilisaient les mêmes formes qu'il s'agisse de la première référenciation d'un ensemble de référents ou de ses mentions subséquentes au sein du récit. En effet, si lors de la première mention, les enfants recourent davantage à des formes fortes nominales, éventuellement inscrites dans une structure disloquée ou clivée, cela signifie qu'ils tendent à marquer discursivement un contraste avec ce qui précède. À l'inverse, un recours massif à des formes pronominales dès la première mention de la fusion indiquerait une forme de continuité avec les différents antécédents.

\begin{tabular}{|l|r|r|r|r|r|}
\hline & \multicolumn{1}{|l|}{ PROPERS } & \multicolumn{1}{l|}{ DISLO } & \multicolumn{1}{l|}{ NOM } & \multicolumn{1}{l|}{ AUTPRO } & \multicolumn{1}{l|}{ CLIV } \\
\hline RFPR & $70,20 \%$ & $19,21 \%$ & $2,65 \%$ & $6,62 \%$ & $1,32 \%$ \\
\hline RFSC & $71,08 \%$ & $22,89 \%$ & $3,61 \%$ & $1,20 \%$ & $1,20 \%$ \\
\hline Total & $\mathbf{7 0 , 5 1} \%$ & $\mathbf{2 0 , 5 1} \%$ & $\mathbf{2 , 9 9} \%$ & $\mathbf{4 , 7 0} \%$ & $\mathbf{1 , 2 8} \%$ \\
\hline
\end{tabular}

Tableau 1 - Choix des formes pour la première fusion et les fusions subséquentes d'un groupe de référents 
Le tableau 1 indique que l'ensemble des enfants recourent majoritairement aux pronoms lorsqu'ils procèdent à une fusion, peu importe sa position dans le récit. En effet, on relève 70,20 \% de pronoms personnels lors de la première fusion dans le récit (RFPR) et 71,08 \% lors des fusions subséquentes (RFSC). Si on ajoute les autres types de pronoms respectivement présents à hauteur de $6,62 \%$ en première fusion et $1,20 \%$ en fusion subséquente, cela fait $76,82 \%$ de formes pronominales lors des premières fusions et $72,28 \%$ lors des fusions subséquentes. Les formes pronominales font donc l'objet d'un usage massif pour exprimer les fusions à tout moment du récit. Après les pronoms, les dislocations du nom et du pronom sont les formes les plus fréquentes en fusion. Là encore, les enfants y recourent de façon équivalente qu'il s'agisse de la première fois que les référents sont regroupés sous une même expression référentielle ou non. Il n'y a donc pas de différence entre la référenciation première d'une fusion et les fusions subséquentes des mêmes groupes de référents dans le récit. De manière générale, la forte propension à choisir des formes pronominales pour les fusions indique que les enfants les construisent en continuité avec les différentes chaînes référentielles qu'elles regroupent.

\subsubsection{Distribution des formes selon l'âge}

Si le pronom personnel est la forme la plus fréquemment utilisée par tous les enfants lorsqu'il s'agit de regrouper au sein d'une seule expression référentielle des référents préalablement isolés, on note tout de même des différences selon le groupe d'âges. La figure 1 montre que tous les enfants recourent en très faible proportion aux formes nominales, cette observation peut paraître logique dans la mesure où la spécificité des noms les rend meilleurs candidats à la mention de référents isolés. Cela dit, l'exemple [1], présenté en introduction, montre que cet usage n'est pas marginal lorsque la fusion n'apparait pas en continuité directe avec les référents dont elle découle. De même, les structures clivées, par leur fonction de mise en relief d'un élément (Hedberg, 2000; Lambrecht, 2001), pourraient être moins fréquentes lorsqu'il s'agit justement de rassembler plusieurs éléments précédemment évoqués sous un même objet de discours.

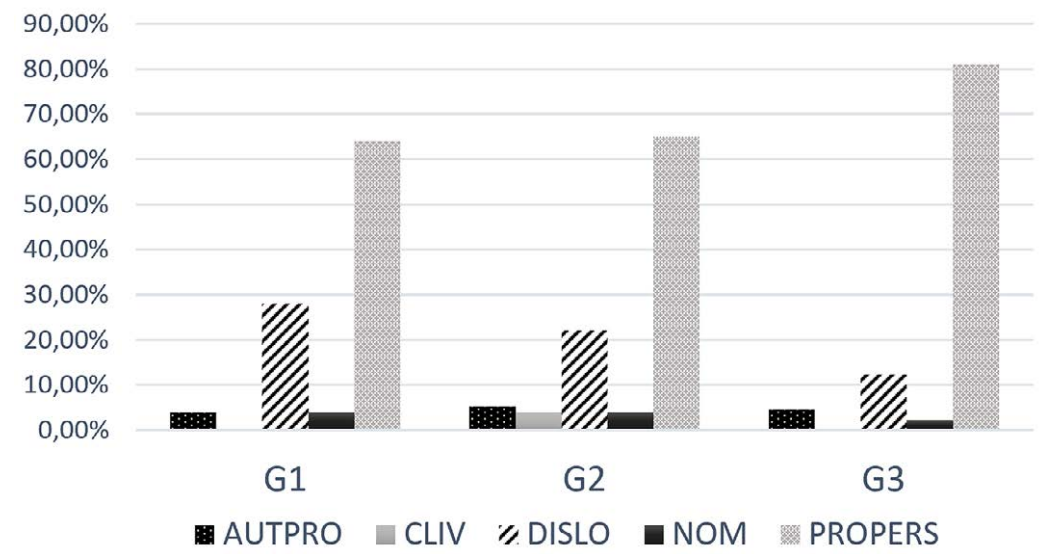

Figure 1 - Choix des formes en fusion selon le groupe d'âges 
Les principales différences entre les trois groupes d'âges quant aux formes utilisées en fusion concernent l'usage des pronoms personnels (Kruskal-Wallis rank sum test, $p=0,02$ ) et des dislocations (Kruskal-Wallis rank sum test, $p=0,06$ ). Les enfants du groupe 3 utilisent en effet significativement plus de pronoms personnels que les plus jeunes des groupes 1 et 2 (Kruskal-Wallis rank sum test, $p=0,01)$. Pour ce qui est des dislocations, seule la différence entre le groupe 1 et le groupe 3 est significative (Kruskal-Wallis rank sum test, $p=0,02$ ). Cela signifie que les enfants de 7-8 ans utilisent davantage les pronoms personnels que les enfants des deux autres groupes. D'autre part, même si l'usage de pronoms personnels est important chez les plus jeunes de 5 et 6 ans, ils utilisent davantage de dislocations que les plus grands en fusion. Ce résultat peut paraitre surprenant dans la mesure où de nombreuses études sur la référence en récit ont justement montré que les récits des jeunes enfants de 4-5 ans étaient marqués par une suite de pronoms personnels, y compris pour passer d'un référent à l'autre, rendant la référence ambiguë pour l'interlocuteur (De Weck, 1991; Hickmann, 2002; Jisa, 2000 ; Karmiloff-Smith, 1985 ; Serratrice, 2013). Cela dit, une revue détaillée de la formation des dislocations dans le corpus, révèle que les enfants recourent presque exclusivement à des dislocations de pronoms lors des fusions, comme dans l'exemple [3]. Seules quelques dislocations de syntagmes nominaux sont à relever en fusion dans le groupe des plus jeunes qui, rappelons-le, est aussi le groupe qui présente le plus fort recours aux formes disloquées (voir le tableau 2). Toutes les autres dislocations en fusion dans le corpus sont des dislocations du pronom tonique ou d'autres types de pronoms (numéraux ou indéfinis), ce qui amène à considérer que le phénomène est assez proche de ce que montre la littérature, les jeunes enfants recourant abondamment à des formes pronominales, qu'il s'agisse de formes clitiques ou de formes fortes, en dislocation.

[3] Cédric, 6;02, G1

ENF6 le renard <il a ch(assé)> [/] il a chassé une poule.

ENF7 et $\mathrm{i}(\mathrm{l})$ court.

ENF8 et l'ours i(l) [/] i(l) [/] i(l) montre du doigt.

ENF9 il va dans la forêt et eux i(l)s sont en train de [/] de [/] de l(e) poursuivre.

Néanmoins, comme le montre l'exemple tiré du récit de Cédric en [3], la dislocation peut être un moyen d'assurer la fonction d'alternance en discours (De Cat, 2007), dans la mesure où la fusion ne fait pas directement suite à la dernière mention d'un des animaux du groupe. En effet, la connaissance du support imagé et la suite de l'énoncé permettent d'interpréter que le pronom «il» en ENF9 renvoie au renard, la mention de l'ours («l'ours il») en ENF8 constitue ainsi l'antécédent le plus proche de la fusion («eux ils») mais n'est pas immédiatement suivie de ladite fusion. Ce contexte pourrait expliquer un plus fort recours aux dislocations: elle sert ici à exprimer un contraste vis-à-vis du pronom «il» qui précède immédiatement dans le même énoncé. On peut donc se demander si les plus jeunes enfants ne seraient 
pas plus sujets à produire des fusions en contexte de concurrence et à distance avec un autre référent, ce qui explique leur plus fort recours à des structures disloquées. À l'inverse, les plus grands pourraient les privilégier en continuité directe avec les antécédents, limitant ainsi les possibilités d'ambiguïtés et leur permettant de faire plus souvent usage du pronom personnel.

\begin{tabular}{|l|r|r|r|}
\hline & G1 & G2 & \multicolumn{1}{l|}{ G3 } \\
\hline DISLO PRONOM & $38,78 \%$ & $34,69 \%$ & $22,45 \%$ \\
\hline DISLO NOM & $4,08 \%$ & $0,00 \%$ & $0,00 \%$ \\
\hline TOTAL & $42,86 \%$ & $34,69 \%$ & $\mathbf{2 2 , 4 5 \%}$ \\
\hline
\end{tabular}

Tableau 2 - Distribution des dislocations du pronom et des dislocations du nom en fusion dans chaque groupe

\subsubsection{Relation à l'antécédent}

\subsubsection{Contexte d'apparition des fusions}

La fréquence des pronoms personnels en fusion étant élevée, en particulier chez les enfants les plus grands, on peut supposer que c'est parce que les fusions apparaissent le plus souvent en continuité avec le ou les antécédents, soit au niveau de la fonction syntaxique, comme en [4] et [5], soit au niveau proximal. Dans ce dernier cas, la fusion est aussi souvent en continuité syntaxique avec un autre de ses antécédents puisqu'elle regroupe à la fois les éléments en position sujet et en position non-sujet de l'énoncé précédent, comme illustré en [6].

[4] Étienne, 6;04, G2

ENF16 après $<$ (il) $\mathrm{y} a>[/]$ (il) $\mathrm{y}$ a son frère $<$ qui $\mathrm{a}>$ [/] qui est venu.

ENF17 i(l) prend les glaces.

ENF18 après eux trois $\mathbf{i}(1) \mathbf{s}$ mangent les glaces.

[5] Caroline, 7;08, G3

ENF3 et une poule le [/] un lapin et l'ours euh courent après le [/] le renard.

ADU6 $\quad+<0$ [=! hoche la tête].

ENF4 mais là $\mathbf{i}(1) \mathbf{s}$ sont un peu épuisés.

[6] Laura, 7;00, G2

ENF26 après la poule défend le renard.

ENF27 après $\mathbf{i}(1) \mathrm{s}$ se font un câlin.

En [6], le pronom «ils» en ENF27 reprend effectivement l'ensemble des référents de l'énoncé précédent, à savoir la poule et le renard. De plus, le pronom réciproque «se» renforce la relation de coréférence du pronom «ils» avec les deux 
entités mentionnées immédiatement avant. Dans l'exemple [4], la continuité entre l'antécédent et la fusion a lieu sans qu'un autre référent animé ne composant pas la fusion ne s'intercale entre les deux. Dans l'exemple [5], il y a également continuité sur le plan syntaxique, bien qu'un référent animé ait été mentionné en position non-sujet entre la fusion et ses antécédents.

S'il s'avère que les enfants du groupe 1 réalisent davantage de fusions dans des contextes marqués - à l'inverse de ces exemples - par l'alternance, cela pourrait expliquer leur plus forte tendance à recourir à des structures disloquées. Ce qui est identifié ici comme un contexte d'alternance correspond soit à une fusion qui ne fait pas immédiatement suite à l'antécédent et qui, de surcroît, est marquée par un changement de fonction par rapport à celui-ci, soit à une fusion séparée de son antécédent par au moins un autre référent animé occupant la même fonction syntaxique comme dans les exemples [1] et [3].

La figure 2 montre la part de fusions apparaissant dans chacun des contextes susmentionnés pour les trois groupes. Si les plus jeunes enfants du groupe 1 semblent effectivement réaliser légèrement plus de fusions en contexte d'alternance que les enfants des deux autres groupes, les différences ne sont pas significatives. Ainsi, il n'y a pas de différence selon l'âge en ce qui concerne les contextes dans lesquels les enfants produisent des fusions. Tous effectuent en grande majorité des fusions dans des contextes de continuité comme ceux présentés dans les exemples [4], [5] et [6]. On notera aussi, à la lumière de l'exemple [4], que la dislocation peut avoir pour fonction de spécifier le caractère pluriel de l'expression référentielle lorsque le verbe ne suffit pas à assurer ce rôle. En effet, si Étienne avait seulement utilisé un pronom personnel lors de la fusion, il y aurait eu ambiguïté dans la mesure où la forme du verbe manger à l'oral est identique au singulier et au pluriel à la troisième personne. Ici, la dislocation sert à indiquer que l'expression référentielle, bien qu'en continuité avec le référent précédemment mentionné, ne renvoie pas uniquement à celui-ci. De fait, l'antécédent pourrait aussi constituer le concurrent de l'expression référentielle fusionnée à laquelle il laisse place en discours, en particulier lorsqu'il est seul à assumer la fonction d'antécédent au sein de l'énoncé précédent, comme dans le récit d'Étienne. Cependant chez les enfants du groupe 1, on trouve plusieurs exemples d'usages de dislocation dans des contextes de continuité où les antécédents étaient tous présents dans l'énoncé précédent et la forme verbale suffisait à exprimer le pluriel (voir l'exemple [7]).

[7] Maxence, 5;10, G1

ENF15 et l'ours le lapin et le coq $+\ldots$

\%com: ENF pointe successivement les référents qu'il mentionne

ADU10 mh-mh +..?

ENF16 +, v(ont) [/] vont à la recherche de la poule!

ENF17 une fois dans la forêt profonde les animaux i(l)s sont fatigués. 


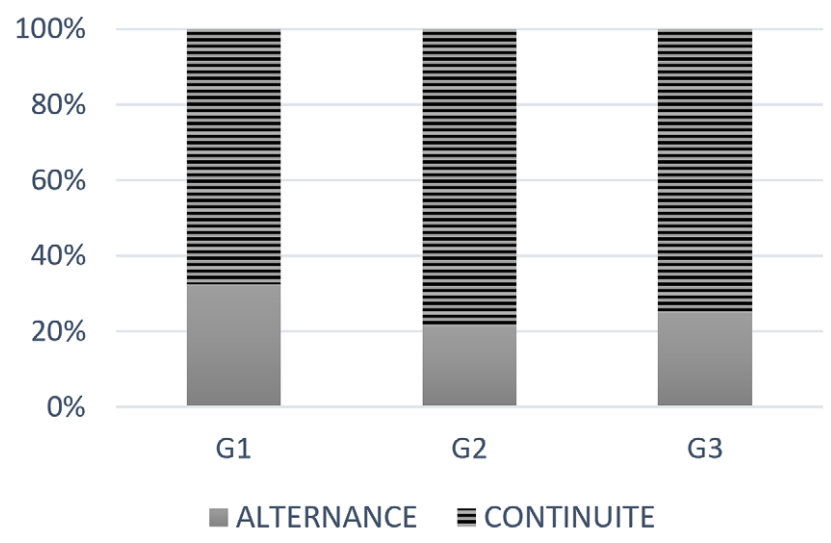

Figure 2 - Continuité et alternance des fusions par rapport à l'antécédent

\subsubsection{Choix des formes en continuité et en alternance}

La comparaison des formes utilisées en fusion selon qu'il y ait alternance ou continuité avec l'antécédent semble indiquer que tous les groupes d'enfants utilisent des formes différentes selon les contextes (voir figure 3). En effet, en continuité, les trois groupes utilisent une majorité de pronoms alors que leur fréquence se réduit au profit des dislocations et, dans une moindre mesure, des noms quand il y a alternance. Cependant, les enfants des groupes 1 et 2 sont les seuls à utiliser significativement moins de pronoms personnels en alternance qu'en continuité (Kruskal-Wallis rank sum tests, $p=0,06$ pour le groupe 1 et $p=0,07$ pour le groupe 2 ). Pour les enfants du groupe 3 , la fréquence de pronoms personnels étant relativement élevée en alternance, la différence entre les deux contextes n'est pas significative. À nouveau, ce résultat peut être perçu comme allant à l'encontre de ce qui a déjà été dit sur le développement de la référence en récit, dans la mesure où les récits d'enfants plus jeunes sont normalement marqués par des difficultés à exprimer l'alternance entre les référents (Bamberg, 1986; Karmiloff-Smith, 1985; Serratrice, 2013).

$\mathrm{Si}$ à tous les âges, on observe des cas de fusion pour lesquels il est difficile d'identifier clairement à quels référents renvoie l'expression référentielle au pluriel, ce n'est pas particulièrement plus fréquent chez les enfants du groupe 3 . Pourtant, leur tendance à privilégier les formes faibles pronominales dans des contextes où plusieurs référents sont candidats à être fusionnés et à ne sélectionner qu'une partie d'entre eux, qui plus est, ceux qui ne sont pas présents de façon la plus proximale ou dans la même fonction syntaxique, devrait entraîner davantage de confusions. À l'image de stratégies déictiques également adoptées par les enfants plus jeunes (Bamberg, 1986; Karmiloff-Smith, 1985), l'ambiguïté possible de la référence de ces pronoms fusionnés en alternance peut être levée par des pointages comme le fait Ramzi dans l'exemple [8]. Sans pointage sur le groupe d'animaux, on pourrait croire que le pronom «ils», en ENF15, renvoie au renard et à la poule (mentionnée par le biais du pronom tonique «elle» qui précède). 


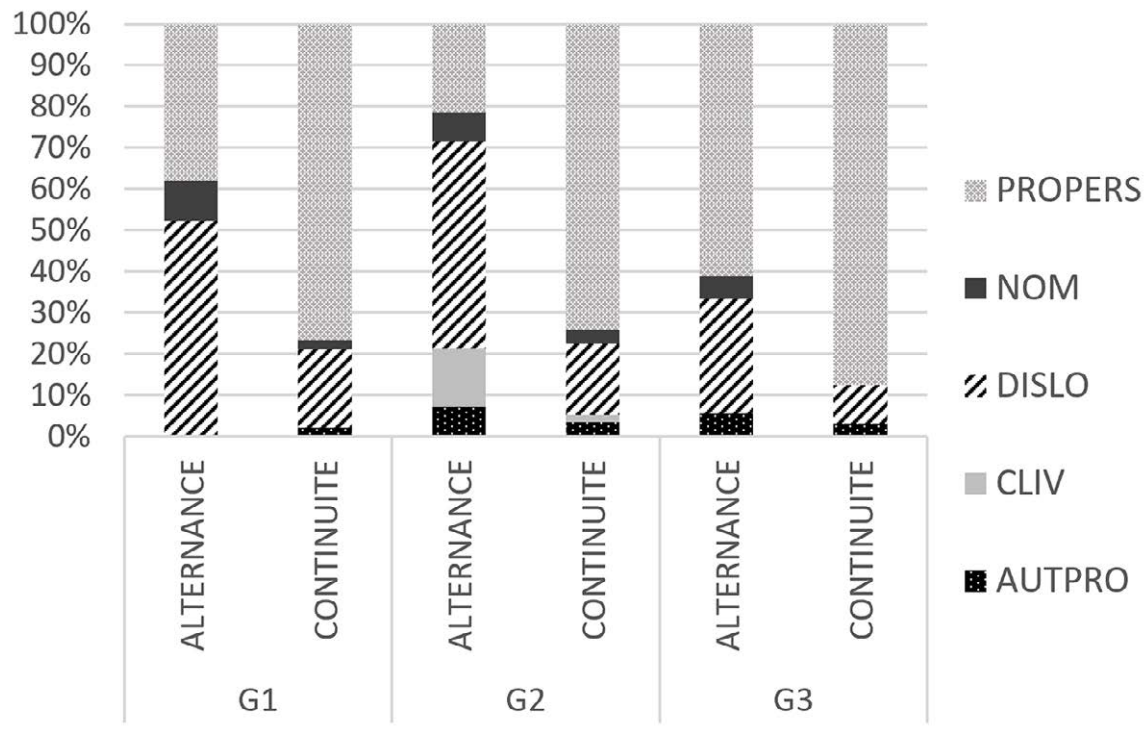

Figure 3 - Choix des formes pour les fusions en alternance et en continuité selon le groupe d'âges

[8] Ramzi, 8;04, G3

ENF13 après comme il était rentré dans 1(e) terrier l'ours il était coincé.

\%com: ENF pose sa main sur le renard puis pointe les galeries

ENF14 et en fait c'était pas pour la manger c'était pour faire une partie d'échecs!

ADU14 ben ouais!

ENF15 après le renard il a dormi avec elle et <i(l)s ont fait un [/] un camping> [/] i(l)s ont campé.

\%com: ENF pose la main sur le renard et la poule et pointe le groupe sur la colline

On observe cependant chez les enfants du groupe 3 des pronoms personnels utilisés en fusion qui, malgré le contexte d'alternance, se reposent habilement sur l'univers discursif pour faire passer les référents de la fonction sujet à une autre position syntaxique tout en restant clairs pour l'interlocuteur, comme dans l'exemple [9]. En effet, ici, la fusion change de fonction syntaxique par rapport à ses antécédents dont elle se retrouve séparée par un autre ensemble d'entités coordonnées assurant la fonction sujet («l'ours, le lapin et le coq»). Mais le choix d'utiliser des noms pour cet ensemble de référents suffit à exprimer le contraste et à rendre le renard et la poule facilement identifiables en tant que candidats à être fusionnés en position objet. Dans ce cas-là, la focalisation étant déjà portée sur un élément en position sujet, les candidats potentiels à être repris dans d'autres fonctions sous forme de pronoms sont ceux qui étaient précédemment sous le focus d'attention. 


\section{[9] Driss, 8;04, G3}

ENF7 et le renard et la poule grimpa sur un arbre pour pas qu(e) l'ours le lapin et le coq les voient.

\subsection{Les partitions}

\subsubsection{Distribution des formes selon l'âge}

Contrairement aux fusions, lors des partitions, il y a davantage de différences dans le choix des formes selon qu'il s'agit de la première fois que l'entité est mentionnée seule dans le discours (RFPR) ou d'une reprise ultérieure du référent isolé après avoir fait l'objet d'une fusion (RFSC). C'est pourquoi la figure 4 distingue les premières mentions de référents isolés dans le récit des mentions subséquentes après fusion.

Alors que les formes fortes nominales permettant d'individualiser les référents dominent en première mention pour tous les groupes d'âges, les formes exprimant davantage la progression dans le récit (pronoms et dislocations) sont plus fréquentes lorsque les entités isolées se trouvent mentionnées à nouveau après avoir été fusionnées. Si lors de la première mention d'un référent isolé (RFPR), la fréquence des noms semble diminuer entre le groupe 1 et le groupe 3 , les différences ne sont pas significatives, les conduites étant assez variées d'un enfant à l'autre. De même, les secondes mentions isolées d'un référent après avoir fait l'objet d'une fusion (RFSC) ne montrent pas de différences inter-âges significatives.

Globalement, on observe beaucoup de formes fortes et très peu de formes pronominales lors des partitions. Contrairement aux fusions qui consistent en fait à regrouper plusieurs référents potentiellement en concurrence dans la continuité discursive pour n'en faire qu'une seule entité, les partitions consistent à isoler un membre pour lui associer des propriétés différentes de celles du groupe auquel il était associé jusqu'alors. La partition est donc, par essence, un processus impliquant une catégorisation ou recatégorisation du référent et l'ensemble des référents qui composent le groupe précédemment mentionné sont candidats à en faire l'objet. De ce point de vue, en partition, l'antécédent constitue systématiquement un concurrent aussi. En fusion, on a vu que l'antécédent pouvait être un concurrent et entraver la clarté de l'expression référentielle dans des situations très spécifiques liées à la proximité de certains antécédents par rapport à d'autres et au choix de la forme verbale. La mention d'un élément isolé au sein d'un groupe d'entités nécessite donc de recourir davantage à des formes fortes, plus spécifiques (noms et dislocations du nom) ou exprimant un contraste (dislocations et clivées), pour être transparente du point de vue de l'interlocuteur. Les partitions fonctionnent assez différemment des fusions dans la dynamique du discours: elles semblent davantage intervenir en discontinuité avec l'antécédent (les chaînes référentielles fusionnées dont elles découlent) que d'assurer la poursuite de celui-ci. 


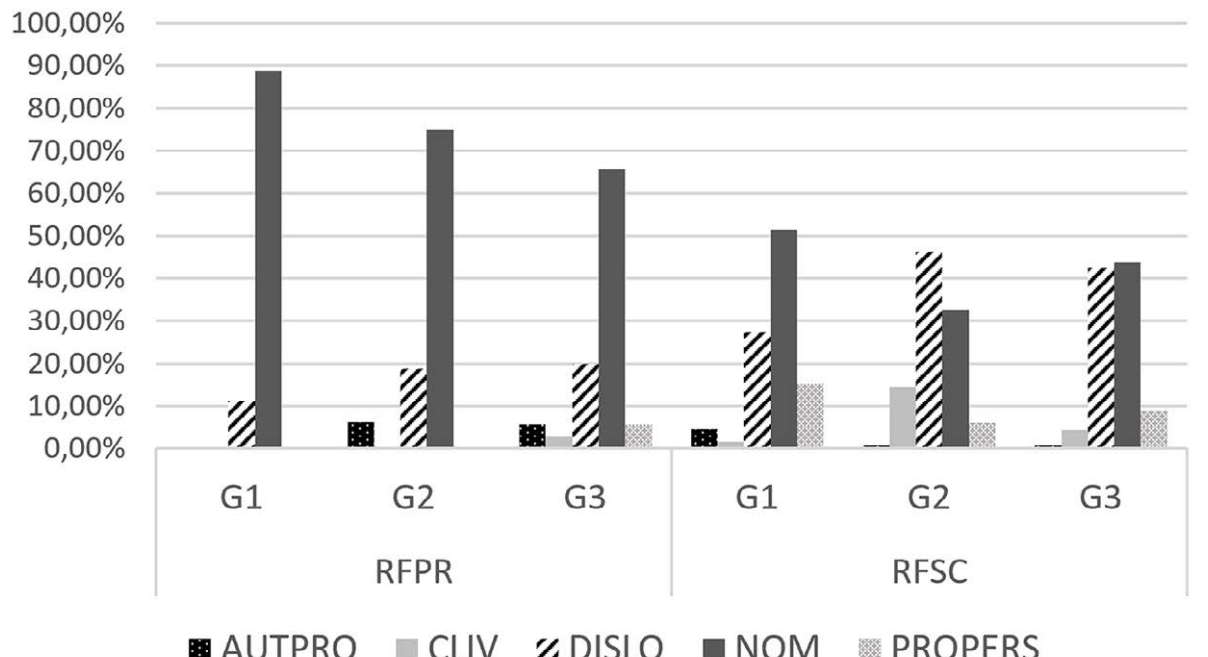

Figure 4 - Choix des formes en partition selon la position sur la chaîne référentielle

\subsubsection{Relation à l'antécédent}

\subsubsection{Contexte d'apparition des partitions}

L'observation du contexte d'apparition des partitions en relation avec l'antécédent confirme la relative autonomie de la partition vis-à-vis de son antécédent. En effet, à la différence des fusions, les partitions ont majoritairement lieu en contexte d'alternance par rapport à l'antécédent (voir figure 5).

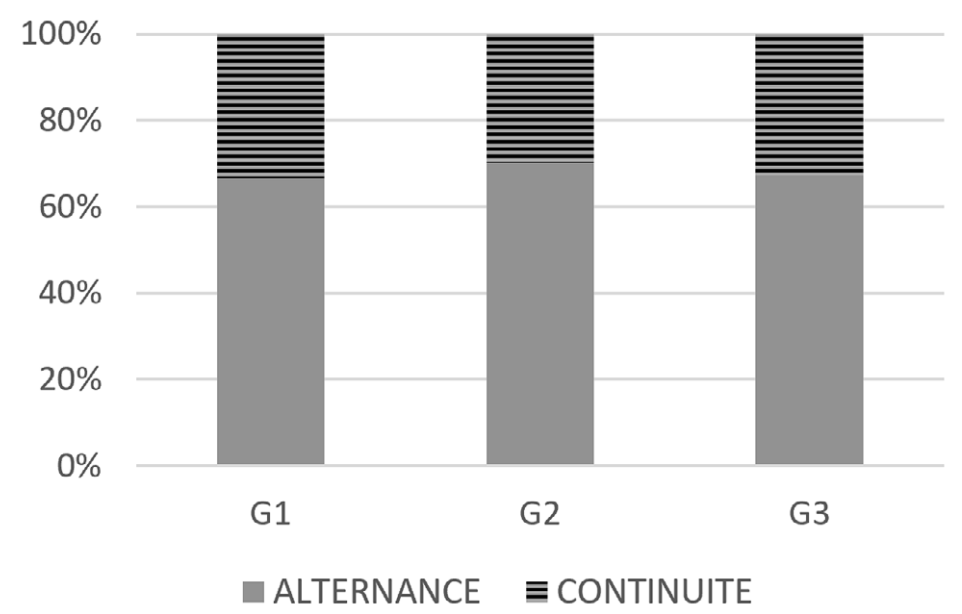

Figure 5 - Continuité et alternance des partitions par rapport à l'antécédent 
Alors qu'en contexte de fusion le taux d'alternance oscillait entre 20 et $30 \%$ selon les groupes, ici il est proche de $70 \%$ pour les groupes 1 et 3 et atteint ce taux pour les enfants du groupe 2. Cela veut dire que les partitions sont, la plupart du temps, exprimées avec une certaine distance et/ou sans assurer le maintien dans la même position syntaxique entre l'antécédent et la partition. Ce résultat explique dans un premier temps la forte présence de formes fortes en partition (voir figure 4), la part importante d'alternance limitant par définition le recours à des formes pronominales. Aussi, cela indique que la partition est moins solidement ancrée dans la chaîne référentielle dont elle découle que ne l'est la fusion.

\subsubsection{Choix des formes en continuité et en alternance}

Pour l'étude des formes selon le contexte dans lequel les phénomènes de partition apparaissent, les analyses se sont concentrées sur les mentions isolées d'objets de discours qui avaient déjà été mentionnés précédemment (RFSC). En effet, lors des premières mentions, le référent n'ayant jamais été mentionné seul dans le discours, il est très peu accessible et les enfants recourent logiquement de façon importante aux syntagmes nominaux pour le désigner, qu'il y ait alternance ou continuité. Ainsi, la distribution des formes choisies en alternance et en continuité vis-à-vis de l'antécédent illustrée sur la figure 6, ne porte que sur les contextes où le référent a déjà été mentionné seul avant de faire l'objet d'une fusion puis d'être à nouveau isolé dans le discours.

La figure 6 indique des différences dans la distribution des formes entre les groupes, en particulier concernant l'usage des pronoms personnels en alternance (Kruskal-Wallis rank sum test, $p=0,08$ ). Une comparaison inter-groupe plus approfondie révèle que la différence est plus importante entre le groupe 3 et les enfants plus petits du groupe 1 (Kruskal-Wallis rank sum test, $p=0,03$ ). Les enfants du groupe 1 utilisent donc plus de pronoms personnels lorsqu'ils produisent une partition qui n'est pas en continuité immédiate avec l'antécédent. Cette fois, le résultat s'avère assez similaire aux phénomènes observés dans les études comparatives sur le développement de la référence en récit (Bamberg, 1986; Jisa, 2000; KarmiloffSmith, 1985; Serratrice, 2013), les enfants entre 4 et 6 ans utilisant fréquemment des formes pronominales dans des contextes de première mention ou en réintroduction, avec de nombreux référents en concurrence qui auraient nécessité l'usage d'une forme forte. En effet, dans l'exemple [10], l'enfant procède à deux partitions successives en utilisant des formes pronominales. De ce fait, le pronom «il» en ENF27 nécessite de se rapporter au support imagé pour être interprété comme une partition de l'expression référentielle en ENF25 et pas du pronom «ils» en ENF26, lui-même une partition qui renvoie au groupe d'animaux.

[10] Baptiste, 6;00, G1

ENF25 et là $<\mathrm{i}(1) s$ sont $>[/] \mathrm{i}(1) \mathbf{s}$ sont tous amis!

ENF26 et là i(l)s sont partis avec le bateau.

ENF27 et il est resté avec la poule 


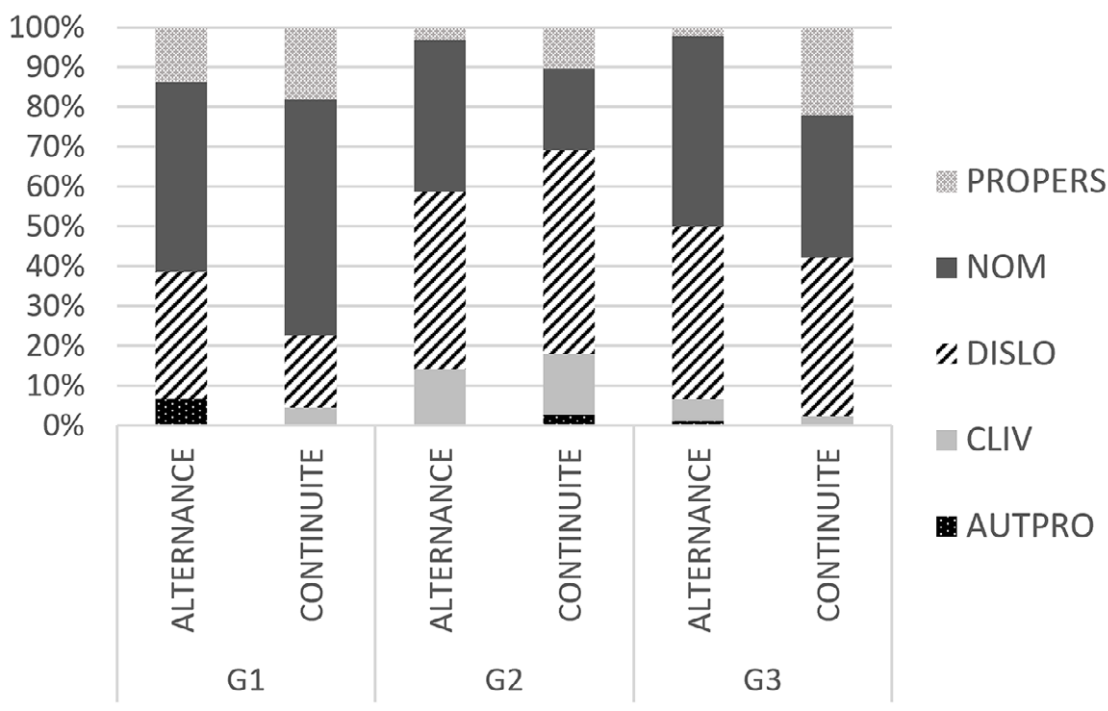

Figure 6 - Choix des formes pour les partitions en alternance et en continuité selon le groupe d'âges

Dans l'exemple suivant, en [11], la partition intervient même à une certaine distance de l'antécédent et passe de la fonction sujet à la fonction objet. Alors que la mention du renard et de la poule constituait le thème de l'énoncé en ENF28 («eux ils»), l'un des deux éléments se retrouve en position objet sous la forme d'un pronom objet («l'» en ENF33) à trois énoncés d'intervalle. Ainsi, on ne sait pas vraiment qui de la poule ou du renard se retrouve dissocié à cet endroit du récit.

\section{[11] Léa, 6;01, G1}

ENF28 et eux i(l)s étaient tranquilles.

\%com: ENF pointe le renard et la poule dans la barque

ENF29 après eux $\mathrm{i}(1) \mathrm{s}$ sont rentrés.

ENF30 et $\mathrm{i}(1) \mathrm{s}$ étaient tout mouillés.

ENF31 +, trempés!

ENF32 et du coup $<\mathrm{i}(1)$ s l'ont $>[/] \mathrm{i}(\mathrm{l}) \mathrm{s}$ sont rentrés.

\%com: ENF pose la main sur le groupe d'animaux

ENF33 et finalement ils l'ont laissé [= ? laissée] tranquille

Dans un second temps, lorsqu'au sein d'un même groupe d'âges on compare les conduites en alternance et en continuité, on note que les enfants des groupes 2 et 3 recourent très peu aux formes faibles pronominales en alternance alors que dans le groupe 1 , on trouve une proportion similaire de pronoms personnels dans les deux contextes. Les enfants du groupe 3 sont cependant les seuls à utiliser significativement plus de formes pronominales en continuité qu'en alternance 
(Kruskal-Wallis rank sum test, $p=0,008$ ). Ainsi, les enfants plus grands présentent relativement peu d'usages comme en [10] et en [11] consistant à dissocier un référent du groupe à distance avec un pronom personnel, ils semblent au contraire privilégier les pronoms lorsque la continuité est immédiate entre la mention du groupe et celle d'une de ses entités. Cependant, comme évoqué précédemment, le fait de passer de la mention d'un groupe d'individus à un individu isolé a pour effet de mettre en concurrence toutes les entités qui composent le groupe, de ce fait, même en continuité, l'usage de pronoms personnels en partition pourrait s'avérer ambigu pour l'interlocuteur. Or, plusieurs exemples tirés du groupe 3 donnent à voir des cas où le recours à des formes pronominales en partition n'est pas ambigu dans la mesure où tous les membres du groupe sont partitionnés dans le même énoncé, ce qui permet à l'interlocuteur d'inférer quel référent est repris sous forme pronominale. C'est le cas en [12], où l'enfant use en plus des caractéristiques grammaticales de la poule, qui est un référent féminin, pour la reprendre en position sujet sous la forme du pronom «elle», et ainsi neutraliser la concurrence avec le renard avec qui elle était regroupée en ENF24.

\section{[12] Ingrid, 7;09, G3}

ENF24 puis après $\mathbf{i}(1)$ s disent pardon.

\%com: ENF pose la main sur le renard et la poule

ENF25 après elle fait un bisou au renard!

L'exemple [13] montre un fonctionnement similaire, les deux éléments partitionnés sont repris sous forme pronominale dans l'énoncé suivant. L'usage d'un pronom objet féminin permet de comprendre que le pronom sujet correspond au renard alors que la poule est en position objet en ENF50. Il se pourrait alors, qu'en plus de maitriser la différence pragmatique en termes d'accessibilité dans les contextes d'alternance pour favoriser l'usage des pronoms en continuité, les enfants plus grands soient davantage en mesure de les utiliser dans des situations où ils n'entraînent pas de confusions malgré la concurrence avec l'antécédent.

[13] Lola, 8;01, G3

ENF47 et $\mathrm{i}(\mathrm{l}) \mathrm{s}$ voyent le renard avec la poule tranquillement en train $\mathrm{d}(\mathrm{e})$ rigoler.

ENF48 $0[=!$ rit $]$ euh là alors là $\mathrm{xxx}+\ldots$

\%com: ENF parle en riant et marmonne

ADU30 hein?

ENF49 ouais parce que là en fait $\mathbf{i}(1)$ s sont tous les deux $0[=!$ rit $]$ !

\%com: ENF pointe le renard et la poule puis tourne la page

ENF50 imagine finalement là à la fin i(l) la mange $0[=!$ rit $]$ !

\%com: ENF pointe le renard

ADU31 0 [=! rit $]$ na:n?

ENF51 $\quad+<$ ben nan quand même il la mange pas! 


\section{Discussion et conclusion}

Ces résultats apportent dans un premier temps des arguments en faveur du fonctionnement distinct des processus de fusion et de partition en discours. En effet, le premier phénomène semble davantage relever de la continuité des chaînes référentielles précédemment évoquées. Les fusions présentent ainsi un fonctionnement assez proche de celui des référents évolutifs, en ce sens que les formes pronominales ne servent pas à reprendre l'identité matérielle de l'antécédent mais servent plutôt à exprimer la coréférence avec cet antécédent enrichi de nouvelles propriétés (Charolles et Schnedecker, 1993). Les partitions, quant à elles, marquent une rupture et nécessitent un recours à des formes plus fortes que les formes pronominales qu'on trouve massivement en fusion. En outre, si pour les fusions, la composante la plus proche dans le discours peut s'avérer être à la fois un antécédent et un concurrent dans certaines situations, pour les partitions, l'antécédent apparait plus systématiquement comme un concurrent dans le discours. Cependant, comme le soulignent Schnedecker et Landragin (2014), les partitions et les fusions fonctionnent bien par mouvement symétrique en discours dans la mesure où, idéalement, la mention de toutes les parties immédiatement avant la fusion, comme en [5], en [6] ou en [7], a pour effet de clarifier à qui celle-ci renvoie exactement alors qu'à l'inverse, la mention de toutes les parties directement après le tout, comme en [12] et [13], permet d'identifier plus facilement chacune des entités partitionnées. Seulement, à l'oral et en particulier avec des locuteurs enfants racontant un récit à partir d'un support qui leur est présenté en simultané, fusions et partitions ne se construisent pas systématiquement selon ces ressorts pragmatiques. Il semblerait que la pluralité des antécédents en fusion, même s'ils ne sont pas à proximité directe, les rende plus accessibles et permette davantage le recours à des formes brèves exprimant la progression dans le discours (Ariel, 1991). En cela, les antécédents des fusions constituent plus souvent de vrais antécédents qui laissent place à des reprises sous la forme de pronoms personnels, que des concurrents sur le plan référentiel. À l'inverse, en partition, les formes utilisées semblent moins assurer la progression de l'antécédent qu'entrer en concurrence avec lui, expliquant alors la forte présence de noms et de dislocations pour envisager un contraste avec l'expression référentielle dont la partition dérive. Du fait de leurs caractéristiques, les fusions pourraient ainsi, même quand elles introduisent un nouvel objet de discours, ne pas avoir à revêtir les caractéristiques discursives d'une première mention, participant davantage à la progression des différentes chaînes référentielles qu'elles regroupent. Le choix des formes en fusion semble en effet être motivé par des facteurs pragmatiques de proximité et de continuité fonctionnelle avec l'antécédent. Les partitions, quant à elles, fonctionnent davantage de façon autonome, par distinction avec l'antécédent, et semblent opérer un retour en arrière, revenant à la mention isolée d'un référent qui avait évolué en fusion. Dans le cas de premières mentions d'un référent découlant d'un groupe partitionné, on trouve des phénomènes similaires aux premières mentions en discours en général (Charolles, 2002 ; Corblin, 1987 et 1995). De fait, les partitions opérant la référenciation 
première d'un référent isolé, à la différence de la première référenciation d'une fusion, laissent davantage place à une nouvelle chaine référentielle plutôt qu’à assurer la progression de la chaîne référentielle dont elles découlent.

Dans un second temps, les comparaisons inter-âges montrent que les enfants vont vers un usage de plus en plus massif des pronoms personnels lors des fusions, même lorsque ces dernières n'apparaissent pas dans la continuité directe de l'antécédent. Ils parviennent en effet à associer les formes fusionnées à des verbes dont les spécificités sémantiques et morphologiques dissipent les possibilités d'ambiguïtés liées au choix d'une forme pronominale. Avant 7 ans, les enfants sont plus enclins à utiliser les dislocations qui servent à marquer l'alternance en discours. Cependant, on ne peut pas dire qu'ils recourent davantage à ces formes parce qu'ils font plus de fusions dans des contextes de concurrence entre référents. De plus, dans ces mêmes contextes, les plus grands s'arrangent pour recourir quand même à des formes pronominales le plus souvent. Ainsi, les enfants plus jeunes marquent moins volontiers le phénomène de continuité entre référents seuls et chaînes fusionnées. Cette spécificité constitue peut-être une caractéristique générale des récits de jeunes enfants puisque Karmiloff-Smith (1985) avait déjà noté que les enfants de 4-5 ans avaient tendance à raconter les histoires image par image sans réellement inclure les référents dans la progression d'un récit. On peut également interroger l'impact du niveau de développement linguistique et cognitif dans ces différences entre les plus grands et les plus petits. En effet, les enfants du groupe 3 , dont les conduites en fusion et en partition se distinguent le plus des deux autres groupes, bénéficient de deux années de scolarisation à l'école primaire, accumulant ainsi les apports d'un enseignement explicite de la grammaire et d'une expérience de la lecture dont on sait qu'elle entretient un lien avec le développement du lexique (Ecalle et Magnan, 2006; Sénéchal, 2000). Cette expérience des discours pourrait en partie expliquer les habiletés des enfants plus grands à éviter les ambiguïtés en fusion en s'appuyant sur différents éléments constitutifs du contexte et de l'univers discursif (Schnedecker et Bianco, 1995) et pas seulement sur l'expression référentielle elle-même et son antécédent.

À l'inverse, pour les partitions, les enfants plus grands se distinguent des plus jeunes par une plus faible utilisation des formes pronominales lorsqu'il y a alternance avec un autre référent dans le récit. Lors des partitions en récit, en plus d'être en concurrence avec les autres référents éventuellement mentionnés, le référent se retrouve en concurrence avec l'ensemble des entités avec lesquelles il avait été fusionné. De ce fait, le choix des pronoms personnels dans des contextes d'alternance chez les plus jeunes entraîne un risque de confusion pour l'interlocuteur. Les plus jeunes enfants ne différencient pas non plus les contextes de continuité, quand la partition intervient immédiatement après la dernière mention du groupe, et les contextes d'alternance, lorsqu'un référent étranger au groupe s'est glissé entre la mention du groupe et la mention d'un référent issu de la partition. Ils produisent en effet autant de formes pronominales dans les deux contextes alors que les plus grands les privilégient en continuité. Cette fois, les résultats vont dans le sens des 
observations sur le développement de la référence en récit chez l'enfant (Bamberg, 1986; Jisa, 2000; Karmiloff-Smith, 1985). On peut également se demander si les compétences cognitives de l'enfant, en particulier les capacités à effectuer des opérations concrètes de conservation et d'inclusion, n'entreraient pas en jeu dans les différences inter-âges observées (Meljac et Lemmel, 2007). En effet, fusionner et partitionner des référents au fil du discours nécessite à la fois de conceptualiser que le même référent demeure sous des formes différentes selon qu'il est mentionné groupé ou isolé (conservation), mais l'enfant doit aussi user de ses capacités à inclure et identifier une entité comme constitutive d'un ensemble plus grand pour manier les phénomènes de fusion et de partition en discours. Dans la tradition piagétienne, le stade des opérations concrètes chez l'enfant n'émerge que vers 7 ans (Piaget, 1979; Piaget et Inhelder, 1967). Ainsi, si on s'en tient à la théorie piagétienne, les enfants du groupe 1 se situent davantage dans le stade préopératoire où ils apprennent à effectuer des opérations élémentaires de classification et les enfants du groupe 2 sont à l'interface entre les deux (Piaget, 1936). Cela pourrait expliquer que les enfants du groupe 3 , ayant tous 7 ans révolus lors de l'étude, se distinguent davantage des deux autres groupes.

Ces premières remarques mériteraient d'être comparées avec des données recueillies auprès d'adultes dans les mêmes conditions, les différences observées au sein des conduites narratives des enfants les plus grands de l'étude apportant peut-être des informations sur le fonctionnement général de ces deux phénomènes à l'oral. Avec ce travail sur les enfants, celui sur des adultes pourrait également contribuer à une comparaison des processus de fusion à l'oral avec les phénomènes déjà observés à l'écrit (Schnedecker et Bianco, 1995). En effet, l'instantanéité de l'oral ne permet peut-être pas aux locuteurs de recourir à une multitude de paramètres discursifs de façon aussi précise qu'à l'écrit pour délimiter les référents regroupés sous une expression référentielle au pluriel, mais les usages enfantins semblent indiquer que le choix des formes en fusion s'appuie bien sur différents niveaux de l'univers discursif institué.

\section{Références bibliographiques}

Aksu-Koç, A. et Nicolopoulou, A. 2015. Character Reference in Young Children's Narratives: A Crosslinguistic Comparison of English, Greek, and Turkish. Lingua 155 : 62-84.

Allen, S. E. M., Hughes, M. E. et Skarabela, B. 2015. The Role of Cognitive Accessibility in Children's Referential Choice. In L. Serratrice et S. E. M. Allen (éd.), The Acquisition of Reference. Amsterdam - Philadelphie: J. Benjamins: 123-153.

ArIel, M. 1991. The Function of Accessibility in a Theory of Grammar. Journal of Pragmatics 16 (5) : 443-463.

Arnold, J. E. et Griffin, Z. M. 2007. The Effect of Additional Characters on Choice of Referring Expression: Everyone Counts. Journal of Memory and Language 56 (4): 521-536. 
BAmberg, M. 1986. A Functional Approach to the Acquisition of Anaphoric Relationships. Linguistics 24 (1): 227-284.

Berman, R. A. et Slobin, D. I. (éd.) 1994. Relating Events in Narrative: A Crosslinguistic Developmental Study. Hillsdale: L. Erlbaum.

Charolles, M. 1988. La gestion des risques de confusion entre personnages dans une tâche rédactionnelle. Pratiques 60 (1): 75-97.

Charolles, M. 2002. La référence et les expressions référentielles en français. Paris - Gap: Ophrys.

Charolles, M. et Schnedecker, C. 1993. Coréférence et identité : le problème des référents évolutifs. Langages 112: 106-126.

Corblin, F. 1987. Indéfini, défini et démonstratif: constructions linguistiques de la référence. Genève - Paris: Droz.

CoRblin, F. 1995. Les formes de reprise dans le discours: anaphores et chaînes de référence. Rennes: Presses universitaires de Rennes.

De Cat, C. 2007. French Dislocation: Syntax, Interpretation, Acquisition. Oxford - New York: Oxford University Press.

De Weck, G. 1991. La cohésion dans les textes d'enfants: étude du développement des processus anaphoriques. Neuchâtel: Delachaux et Niestlé.

De Weck, G. et Salazar Orvig, A. 2014. Comment des mères racontent-elles une histoire à leur enfant? Usage des expressions référentielles dans le dialogue mère-enfant. In M. Fossard et M.-J. BÉGUELIN (éd.), Nouvelles perspectives sur l'anaphore: points de vue linguistique, psycholinguistique et acquisitionnel. Berne: P. Lang: 307-357.

Ecalle, J. et Magnan, A. 2006. Des difficultés en lecture à la dyslexie: problèmes d'évaluation et de diagnostic. Glossa 79: 4-19.

FAyol, M. 1983. L'acquisition du récit: un bilan des recherches. Revue française de pédagogie $62: 65-82$.

FAYOL, M. 2000. Comprendre et produire des textes écrits : l'exemple du récit. In M. KaIL et M. FAYOL (éd.), L'acquisition du langage. Paris: PUF. Vol. 2: Le langage en développement. $A u$-delà de trois ans: 183-213.

Hedberg, N. 2000. The Referential Status of Clefts. Language 76 (4) : 891-920.

Hickmann, M. 2002. Children's Discourse: Person, Space and Time across Languages. Cambridge Studies in Linguistics 98. Cambridge - New York: Cambridge University Press.

Hickmann, M., Schimke, S. et Colonna, S. 2015. From Early to Late Mastery of Reference: Multifunctionality and Linguistic Diversity. In L. Serratrice et S. E. M. Allen (éd.), The Acquisition of Reference. Amsterdam - Philadelphie: J. Benjamins: 181-211.

JisA, H. 2000. Increasing Cohesion in Narratives: A Developmental Study of Maintaining and Reintroducing Subjects in French. Linguistics 38 (3) : 591-620.

Jisa, H., Chenu, F., Fekete, G. et Omar, H. 2010. Promoting Patients in Narrative Discourse: A Developmental Perspective. In M. Kail et M. Hickmann (éd.), Language Acquisition across Linguistic and Cognitive Systems. Amsterdam - Philadelphie: J. Benjamins: 161-177.

Karmiloff-Smith, A. 1981. A Functional Approach to Child Language. A Study of Determiners and Reference. Cambridge - Londres: Cambridge University Press. 
Karmiloff-Smith, A. 1985. Language and Cognitive Processes from a Developmental Perspective. Language and Cognitive Processes 1 (1): 61-85.

LAmbert, N. 2016. Les triplés. Miam-miam! Paris: N. Lambert.

Lambrecht, K. 2001. A Framework for the Analysis of Cleft Constructions. Linguistics 39 (3): 463-516.

LANDRAGin, F. 2011. Une procédure d'analyse et d'annotation des chaînes de coréférence dans des textes écrits. Corpus $10: 61-80$. En ligne à l'adresse suivante: https://journals. openedition.org/corpus/2010.

MacWhinney, B. 2000. The CHILDES Project: Tools for Analyzing Talk. Mahwah: L. Erlbaum [3 éd.].

Meljac, C. et Lemmel, G. 2007. Observer et comprendre la pensée de l'enfant avec l'UDN-II: clinique piagétienne dans l'examen psychologique, méthodologie, étude de cas. Paris: Dunod.

Peres Bonnemaison, K. 2018. Anaphore et référence en production écrite: étude de textes narratifs d'élèves de 9 à 11 ans, du CE2 au CM2. Thèse de doctorat. Université Toulouse - Jean Jaurès.

Piaget, J. 1936. La naissance de l'intelligence chez l'enfant. Neuchâtel - Paris: Delachaux et Niestlé.

Piaget, J. 1979. L'épistémologie génétique. Paris: PUF.

Piaget, J. et Inhelder, B. 1967. La psychologie de l'enfant. Paris: PUF.

Rodriguez, B. 1993. Le voleur de poule. Paris: Casterman.

Salazar Orvig, A., Marcos, H., Morgenstern, A., Hassan, R., Leber-Marin, J. et PArÈs, J. 2010. Dialogical Beginnings of Anaphora: The Use of Third Person Pronouns before the Age of 3. Journal of Pragmatics 42 (7): 1842-1865.

SCHNEDECKER, C. 2006. Anaphores prédicatives démonstratives: de la cohésion syntagmatique à la cohérence textuelle. Corela HS-5. En ligne à l'adresse suivante: https://journals. openedition.org/corela/1437.

Schnedecker, C. et Bianco, M. 1995. Antécédents “dispersés” et référents conjoints ou la construction mentale et la reprise pronominale des entités plurielles. Sémiotiques 8: 79-108.

Schnedecker, C. et LANdragin, F. 2014. Les chaînes de référence: présentation. Langages $195:$ : 3-22.

SÉNÉCHAL, M. 2000. Examen du lien entre la lecture de livres et le développement du vocabulaire chez l'enfant préscolaire. Enfance 2: 169-186.

Serratrice, L. 2013. The Role of Number of Referents and Animacy in Children's Use of Pronouns. Journal of Pragmatics 56: 31-42.

Serratrice, L. et Allen, S. E. M. (éd.) 2015. The Acquisition of Reference. Amsterdam Philadelphie: J. Benjamins. 